\title{
The Visualization Legacy of Galileo's Medicean Moons
}

\section{Michael Mendillo}

\begin{abstract}
When Galileo published Sidereus Nuncius in 1610, he introduced the visualization aspects of astronomy. While his portrayals of the Earth's moon utilized his considerable talents in perspective and chiaroscuro drawing, the satellites of Jupiter were mere points of light depicted by star symbols embedded within his printed text. Within fifty years, the Harmonia Macrocosmica (1660) of Andreas Cellarius illustrated different cosmologies that included, when logically appropriate, Jupiter with companions of different sizes orbiting at different distances. Telescopic photographs and satellite images of Io, Europa, Ganymede and Callisto did not appear until the twentieth century. Yet, from the late seventeenth century up to the present time, illustrations, paintings and sculptures of the Galilean moons continued to appear in a remarkable breadth of uses and styles.
\end{abstract}

\section{Introduction}

In Sidereus Nuncius (1610), Galileo introduced to the printed page how astronomical phenomena seen through a telescope can be portrayed visually to the reader. The iconic example is his depiction of the Moon with mountains, valleys and shadows describing it for the first time as a place similar in many ways to what we see on Earth. The impact of this view was no less dramatic than to challenge the long-standing belief that celestial objects beyond the Earth were perfect, crystalline spheres of unchanging characteristics. Holy Scripture had linked this concept of purity to the Immaculate Conception of Mary, specifically described in Revelation 12 as 'a woman clothed with the sun and the moon under her feet, and upon her head a crown of twelve stars'.

Galileo's friend, the artist Cigoli, was the first to incorporate a maculate Moon into Marian imagery (1610-1612), an impact of astronomy upon art that did not become a standard. ${ }^{1}$ Indeed, in the decades following Cigoli's mural in the dome of Santa Maria Maggiore

1 Eileen Reeves, Painting the Heavens, Art and Science in the Age of Galileo (Princeton: Princeton University Press, 1997).

Michael Mendillo, 'The Visualization Legacy of Galileo's Medicean Moons', eds. Nicholas Campion and Rolf Sinclair, Culture and Cosmos, Vol. 16 nos. 1 and 2, 2012, pp. 331-343.

www.CultureAndCosmos.org 
in Rome, Immaculatas produced by Perede (1619) and Velazquez (1619) continued the practice of a translucent Moon. ${ }^{2}$

For his discovery of the moons of Jupiter, Galileo encountered no such controversy from the artistic traditions. Indeed, the Bible was silent about moons beyond our own, and thus his portrayal of them using star symbols and naming them after the Medici brothers in Sidereus Nuncius created no controversy of representation. Their very existence was another matter. If another planet could have moons that accompanied it in its motion, then the objection to Copernicanism that a moving Earth would leave the Moon behind (which was obviously not the case) seemed unfounded.

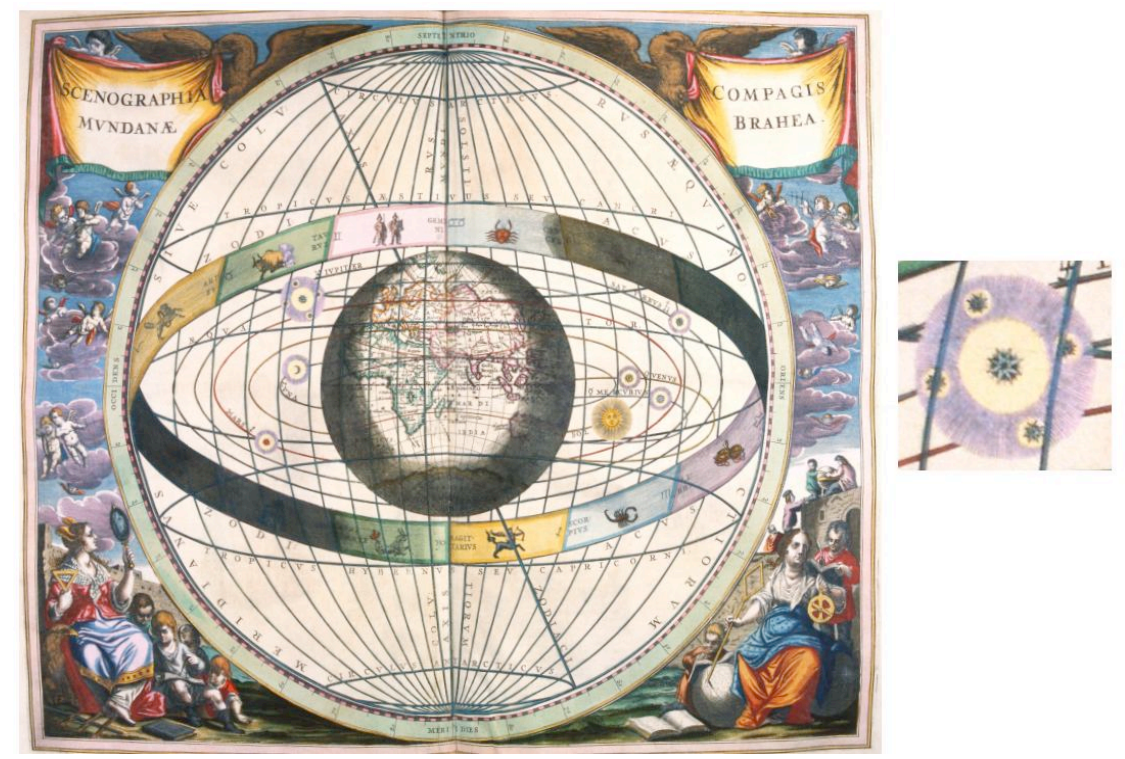

Figure 1. Planisphaerium Braheum from Atlas Coelestis seu Harmonia Macrocosmica ( $1^{\text {st }}$ edition, $2^{\text {nd }}$ printing), Amsterdam, 1662, engraving 16 $3 / 4$ " x 20 5/8" [Mendillo Collection]. Inset shows Jupiter with moons.

This concept of 'a centre of motion can itself be in motion' was not, in fact, a problem for the Church's accepted geocentric cosmology. Tycho Brahe had, most conveniently, introduced a compromise system in which the known planets orbited the Sun, with the Sun and its companions orbiting the Earth-Moon system. Thus a centre of motion (the Sun) could

2 Reeves, Painting the Heavens.

Culture and Cosmos 
be in motion without leaving its brood behind. Depending on one's preference, all one had to do was to add the four Galilean moons to portrayals of either the Copernican or Tychonic systems. Within just fifty years of the publication of Sidereus Nuncius, this was such a standard practice for describing competing cosmologies that it was incorporated into the great visualization compendium of astronomical knowledge produced by Cellarius in $1660 .^{3}$ An example is given in Figure 1.

In this paper, I provide a broad overview of how the Medicean moons were portrayed in the decades and centuries after Cellarius, up to present times.

\section{Late $17^{\text {th }}$ Century and $18^{\text {th }}$ Century Depictions of Jupiter's Moons}

The practice introduced by Cellarius became the standard to follow, namely, to make no judgmental pronouncement about the 'correct' celestial system, but to offer 'options' to consider. In France, atlases showing Four Systems of Cosmology were produced by Alexis Hubert Jaillot (1690) and Nicolas de Fer (1705). Shown in Figure 2 (upper left) is Jaillot's offering of the heliocentric system as described by Descartes. Here we see evidence of modern science. The orbits are more than uniformly spaced concentric circles and the planets are indicated by disks. Jupiter has four moons, also depicted using disks (as Descartes himself did). ${ }^{4}$

Fifteen years later, also in Paris, de Fer offered the systems of Ptolemy, Copernicus, Descartes, and Tycho Brahe juxtaposed for comparison. The fundamental differences between geocentric, heliocentric, and merged geo-heliocentric systems were made explicit. In addition, there are nuances of individuality — such as the spacings between planets and the extents of the firmament of the stars. Notice in particular that de Fer has taken the liberty of updating the plans of Copernicus, Descartes and Tycho by showing discoveries made since their time: the four moons of Jupiter (1610) and the five moons of Saturn which had been discovered by Huygens and Cassini between 1655 and 1684. While the planets are depicted using disks, the moons are all tiny star symbols. Finally, the Ptolemaic system (not shown) remained

3 Michael Mendillo, 'The appearance of the Medicean Moons in 17th Century Charts and Books - How Long Did It Take?', in C. Barbieri et. al (eds.), Galileo's Medicean Moons: Their Impact on 400 Years of Discovery, Proceedings IAU's Symposium No. 269 (2010).

4 Mendillo, 'The Medicean Moons'. 
unchanged, since moons orbiting other planets would not have been consistent with a true geocentric cosmology.
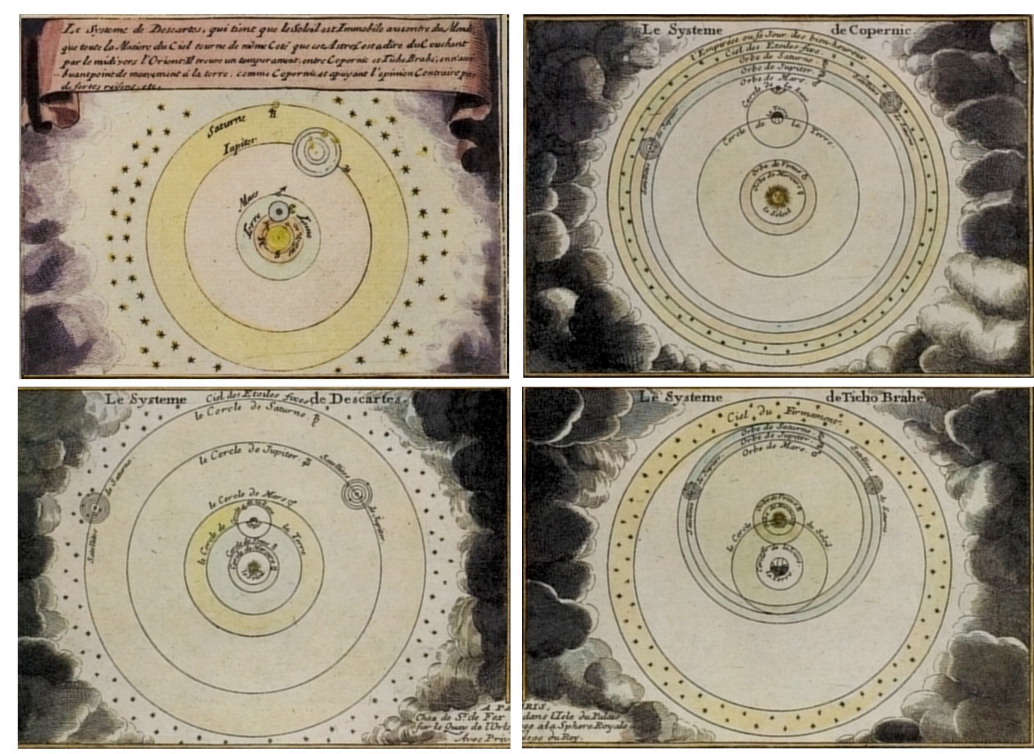

Figure 2. (Upper left) The Descartes' version of Copernicanism, from Four Systems of Cosmology by Alexis Hubert Jaillot (seventeenth century), Paris, c.1690, engraving 5 3/4" x 8 1/4" [Mendillo Collection]. The cosmologies by Copernicus (upper right), Descartes (lower left), and Tycho (lower left), from Four Systems of Cosmology by Nicolas de Fer (1646-1720) with H. van Loon (1649-?), engraver, from Atlas Historique, Paris, 1705, engraving 9 3/8" x 13 1/8" [Mendillo Collection]

\section{Portraying the Jupiter System}

As telescopes improved, emphasis on Jupiter and its four large moons evolved beyond simple updates to the grand cosmological systems under debate. The Jovian system itself became a scientific area of research. This was especially so once Newton's laws of gravity and motion became the accepted explanation for the heliospheric system. The universality of Newtonian physics had a wonderful example in Jupiter, Io, Europa, Ganymede and Callisto being a miniature version of the solar system. While telescopes soon revealed that Jupiter's disk showed banded patterns parallel to its equator, its moons were still too small to be seen as anything other than points of light [the first telescopic picture from Earth to show hints of detail on resolved disks did not occur until early in the 
twentieth century. ${ }^{5}$ The representation method of the moons also evolved to a consensus use of disks - implying that they were physical bodies, presumably not too different from our Moon. Galileo himself had already switched to disks in his Dialogue (1632).

Allain Manesson Mallet was a seventeenth century French military engineer who later became a teacher of mathematics to the pages of Louis XIV. His book Description de l'Universe contains a curious mixture of information on topics ranging from star charts to maps of the ancient and modern world, together with a synopsis of the customs, religion and government of each nation. ${ }^{6}$ Mallet's style for displaying complex information was to do so within a non-threatening context, as shown in Figure 3.

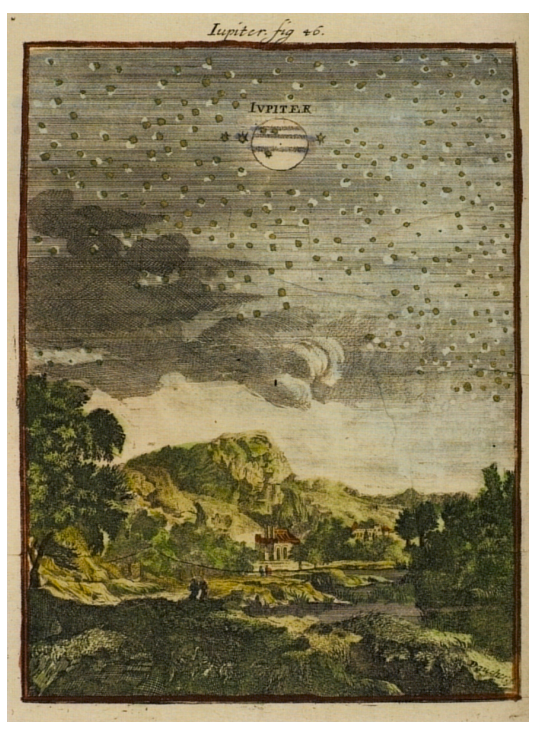

Figure 3. Jupiter, from Description de l'Univers by Allain Manesson Mallet (1630-1706), seventeenth century (German edition), engraving $61 / 8$ " x 4 1/4" [Mendillo Collection]

Here the astronomical message is embedded within a charming rural scene with the clear intent of entertaining the reader while teaching some astronomy. Yet, the actual view is completely unrealistic. What Mallet

5 J. B. Murray 'New Observations of Surface Markings on Jupiter's Satellites,' Icarus, 25, (1975); A. Dollfus, 'History of Planetary Science. The Pic du Midi Planetary Observation Project: 1941-1971,' Planetary and Space Science, 46(8), (1998).

6 Stacy McCarroll, (ed.),Celestial Images: Antiquarian Astronomical Charts and Maps from the Mendillo Collection (Chicago: University of Washington Press, 2005), Exhibition Catalog. 


\section{The Visualization Legacy of Galileo's Medicean Moons}

has done is to show the Jovian system as it would appear through a telescope, inserted within the spatial context of hills, trees, strolling couples and a country villa. Even though it is not nighttime, the sky is richly populated with stars scattered everywhere as small blotches of light. Jupiter appears larger than the mansion below it, with its newly discovered banded atmosphere. Moreover, there is an exuberance of moons! Using six star symbols noticeably different in appearance from the 'normal' background stars, perhaps one (or more) of the three on the disk are meant to inform the viewer about the Great Spot discovered by Cassini in 1660 .

Perhaps the best example of merging telescopic views of Jupiter's moons with landscape painting was achieved by Donato Creti (1671-1749). In 1711 he created a series of 'Astronomical Paintings' to be given to Pope Clement XI. ${ }^{7}$ The eight paintings showed the Sun, Moon, Mercury, Venus, Mars, Jupiter, Saturn and a comet as remarkably prominent objects above pleasant rural scenes, in very much the style of Mallet. The Jupiter image is shown in Figure 4.

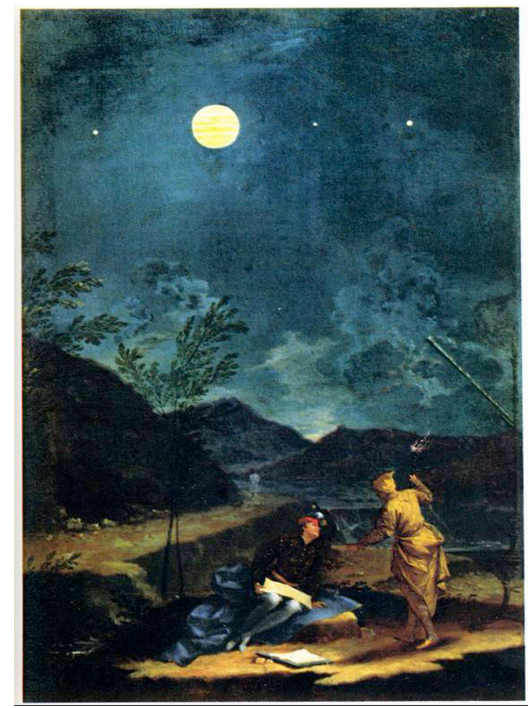

Figure 4. Jupiter, from The Astronomical Observations by Donato Creti, 1711. Oil on canvas, 20 1/4" x 13 3/4" [Pinocoteca, Vatican City]

7 Margaret Frazer, (ed.), The Vatican Collections: The Papacy and Art (New York: The Metropolitan Museum of Art, in association with Harry N. Abrams, Inc., 1982). Exhibition Catalog, 168-171. 
While the format is similar to Mallet's, the important difference is that there are two observers busily at work next to their telescope.

This painting, and its companions, were part of an all-out lobbying effort to convince the Pope to sponsor an astronomical observatory. As such, the paintings are a collaboration between the prominent landscape artist Creti and the miniaturist Raimondo Manzini who inserted the astronomical content. The details are, in fact, impressive. Jupiter has six atmospheric bands spanning its disk, and above the equator is the Great Red Spot (shown in the inverted way most telescopes of the day would have it seen, i.e., the feature is physically in Jupiter's southern hemisphere). The moons are tiny white disks, two to the right and one to the left. The choice not to portray all four moons is an interesting one. Given the possibility that the Pope might actually ask to look through a telescope, setting expectations too high (seeing all four moons on a given night) would not have been wise. For Galileo, it took six nights of observations before he saw all four moons at the same time. ${ }^{8}$ He was thus able to correctly deduce that they pass periodically in front of and behind Jupiter's large disk and are therefore not capable of being seen all the time. The Creti-Manzini collaboration, sponsored by Count Luigi Ferdinando Marsili (1658-1730), succeeded in getting the Pope's financial support for an observatory in Bologna.

\section{Non-Scientific Uses: Galileo and the Corporate Logo}

As a newly discovered phenomenon, Jupiter and its four moons offered a visual appeal beyond the research interests of practicing astronomers. Centuries before the Subaru automobile company chose the Pleiades as its corporate symbol, the iconography introduced by Galileo found its way into the world of business. Appropriately, the book publishers of Europe were among the first to adapt Galileo's sketches of the Jovian system as their corporate logos. Such simple uses of scientific information are in marked contrast to the lavishly produced frontispieces and fully-illustrated title pages of books on astronomy in the seventeenth century. ${ }^{9}$ In Figure 5, the title pages of two books published in 1619 and 1664 offer fine examples of the logo practice.

8 Albert Van Helden, Sidereus Nuncius, or The Sidereal Messenger Galileo Galilei (Chicago: University of Chicago Press, 1989).

9 Inga Elmqvist Soderlund, 'Taking Possession of Astronomy, Frontispieces and illustrated title pages in 17th Century Books on Astronomy' ( $\mathrm{PhD}$ diss., The Center for History of Science, Royal Swedish Academy of Sciences, 2010). 

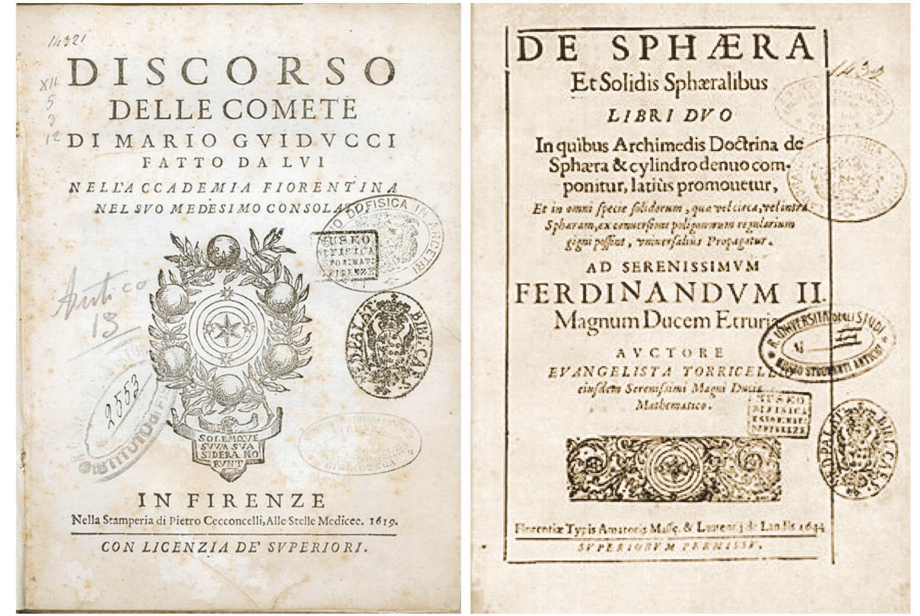

Figure 5. Galilean moon imagery used as printer's logo in $17^{\text {th }}$ Century books. (left) Title page of Discorso Delle Comete by Mario Guiducci (1619) [courtesy of Museo Galileo, Florence, Italy]; (right) Title page of De Sphaera et Solidis Sphaeralibus by Evangelista Torricelli (1644). [courtesy of University of Florence, Florence, Italy via http://www.sba.unifi.it/libri_studio/immagini/2S43_1.jpg]

The Discourse on Comets by Mario Guiducci involved one of the many controversies Galileo is famous for instigating with the Jesuits. In this case, he might have actually been the ghost writer for his student whose name appears as the sole author. ${ }^{10}$ The printing house was in Florence, and so an illustration of the Jovian system is added to the centre of the Medici family crest. The information is representational only, with Jupiter and its four moons depicted as stars viewed face on. In the book by Evangelista Torricellio (1664), the depiction of Jupiter and its moons is essentially identical, but without obvious dedication to the Medici.

Current day uses of Medicean moon imagery in the world of business span an enormous range of applications. In the city where Galileo made his discoveries in 1610 it is perhaps not surprising that many establishments use his name. For example, there is a Hotel Galileo in Padova and its towels are embroidered with a Jovian disk and four star symbols, all in linear fashion, much as they appeared in Sidereus Nuncius. The great planet and its four moons are superimposed upon our

10 'The Galileo Project', Rice University at http://galileo.rice.edu, [accessed 26 January 2011].

Culture and Cosmos 
crescent Moon, even to the point of having some structured detail along the lunar limb (Figure 6).
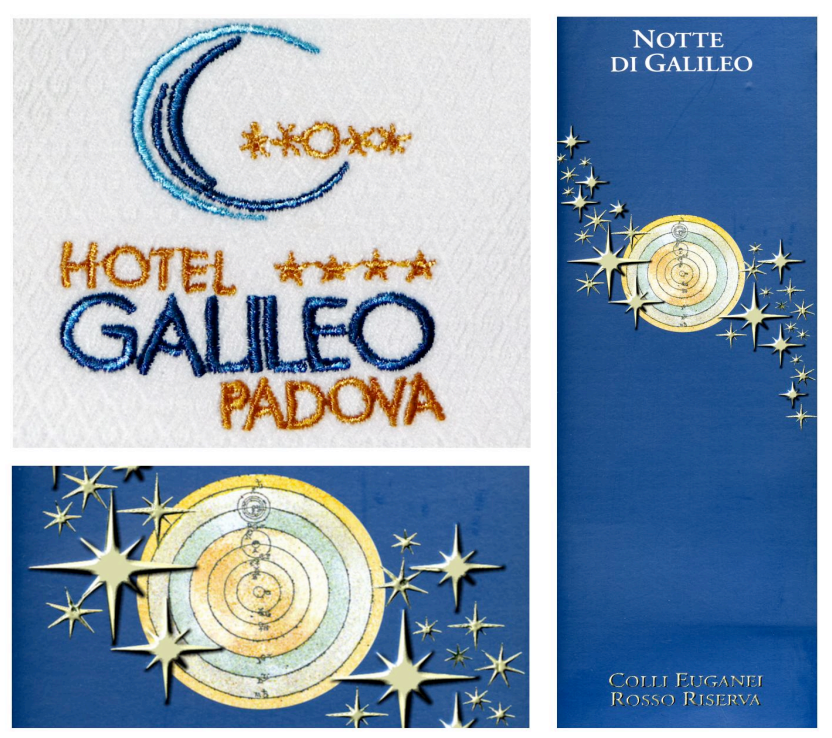

Figure 6. Examples of modern day logos depicting Galileo's discoveries. (top left) towel from Hotel Galileo, Padova; (right and bottom left) Notte di Galileo wine label [photographs by K. Noll (left) and M. Mendillo (right)]

To drive home the point that the moons are not just decoration, they are repeated below to give assurance that the hotel guest is staying in a fourstar hotel. The second image in Figure 6 appears on the label of a red wine produced in the Veneto. Here Galileo's Milky Way is gracefully displayed, together with a representation of our Sun-centred planetary system. In much the same way as shown by de Fer and Jaillot in Figure 2, Jupiter has its four moons.

\section{Galilean Moons in Modern Three-dimensional Objects}

Astronomy can well lay claim to being a visually-dominated field of science. While space and time can be described mathematically in multidimensional ways, it is difficult to show such aspects of Nature in simple visual methods. From the printed books and charts of the past, to the flatscreen, high-definition modes of the computer age, astronomical information is overwhelmingly seen in two dimensions. Video techniques 


\section{The Visualization Legacy of Galileo's Medicean Moons}

and computer animations can portray the full scope of Cartesian, volumetric space, but they present their products upon a flat screen (or with imperfect 3-D glasses). The glorious celestial globes of earlier centuries are seen as three-dimensional objects, but their message is upon a two-dimensional surface. The field of celestial sculpture is a very thin one indeed. Certainly the most famous rendering of the night sky in marble is the Farnese Globe, a second century copy of a Greek sculpture from Hellenistic times, on display at the National Archaeological Museum in Naples, Italy. The constellation figures are without stars, chiselled in high relief from a marble globe. Atlas strains below to hold the heavens upon his broad shoulders. There are no planets, and certainly no Medicean moons, carved in marble to add to Atlas' burden. If the Farnese globe represents the gold medal of astronomical sculpture, there is no consensus whatsoever about the silver medal.

If we turn away from classical art and examine three-dimensional varieties of celestial portrayals, one of the first stops (in modern times) would be with the works of Joseph Cornell (1903-1972). He was most often described as a solitary man, a recluse who found his communications best done via two-dimensional collages and so-called assemblage boxes in three dimensions. A truly remarkable number of his works contained a rich assortment of astronomical information. ${ }^{11}$ This fascination with the celestial domain usually took the form of pasting a portion of a celestial chart or page from an astronomy book on the back wall of his shoe-box-size creations. For example, in a 12 × $21 \frac{1 / 2}{2} 4$ inch box constructed in the early 1950s, behind a small shelf upon which sit five small wine glasses containing various objects, the background is the Systeme de Descartes. ${ }^{12}$ The chart shows Jupiter having four moons, virtually identical to the Descartes panels in de Fer (Figure 2). Thus, Cornell incorporated 'planetary science' into his works, but without an independent visualization method for the Galilean moons.

A mechanical solar system is called an orrery, named after the Earl of Orrery (Charles Boyle), who was a patron of new scientific instrumentation in the early eighteenth century. ${ }^{13}$ Orreries were both

11 Kirsten Hoving, Joseph Cornell and Astronomy, A Case for the Stars (Princeton: Princeton University Press, 2009).

12 Hoving, Joseph Cornell, pp. 161-162.

13 M.J. Babb, The Relation of David Rittenhouse and his Orrery to the University, http://www.library.upenn.edu/exhibits/pennhistory/orrery/orrery.html [accessed 26 January 2011].

Culture and Cosmos 
teaching tools and tributes to the Newtonian Enlightenment ideal of a 'clockwork universe.' One is famously depicted in a painting by Joseph Wright of Derby. ${ }^{14}$ Wound with a key, all of the hidden gears would show how the Universe works via deterministic gravitational dynamics. An orrery that has no moving parts is, by definition, a work of sculpture. A particularly noteworthy modern example appears in Figure 7.

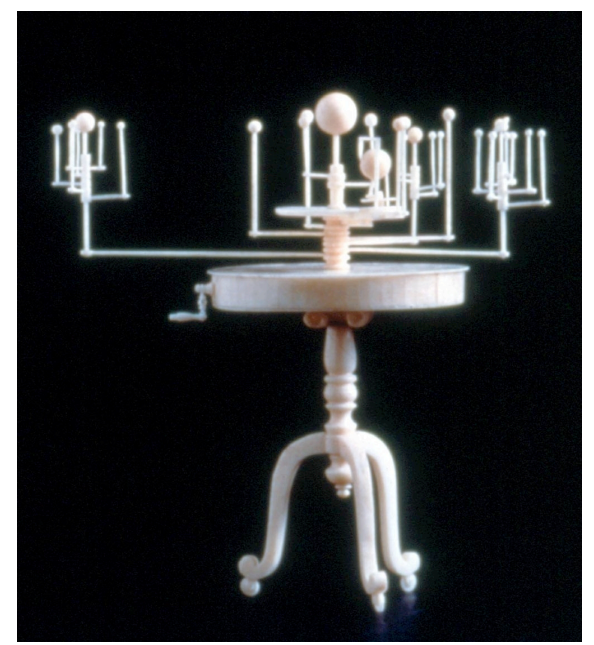

Figure 7. Orrery (1997) by Charles LeDray (1960-), carved from human bone. [private collection; photo courtesy of Institute of Contemporary Art, Boston, MA]

Charles LeDray was born in Seattle (WA) in 1960 and is known as a miniaturist, producing works ranging from tiny hand-stitched clothing to sculpture carved from human bone. His orrery of 1997 is an example of the latter. The full scope of a traditional orrery is shown, with several of the outer planets having their moons, but it is frozen in time and space. The moons Galileo named after the Medici have a particularly strong human connection in this startling portrayal by LeDray.

Contemporary artists specializing in three-dimensional works show no lack of imaginative uses of the Medician moons. Figure 8 contains a selection of pieces done in recent years. In panel (a) is Franceska McCullough's toothpick sculpture entitled Ganymede-Callisto Pod; (b) has Jupiter with its Galilean moons in a ceramic by Paula Rice; (c) shows Galilean moon light fixtures made from repurposed cardboard by Graypants, Inc.; and (d) metallic sculptures of Jovian moons by Mark Castator.

14 Joseph Wright, $A$ Philosopher giving a Lecture on the Orrery in which a lamp is put in place of the Sun, oil on canvas, c. 1766 (Derby Museum and Art Gallery). 
a)

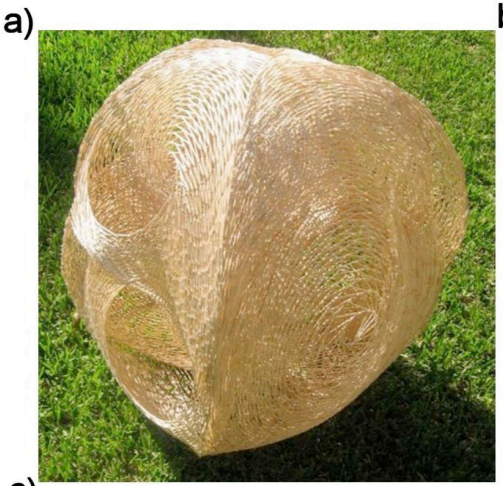

c)

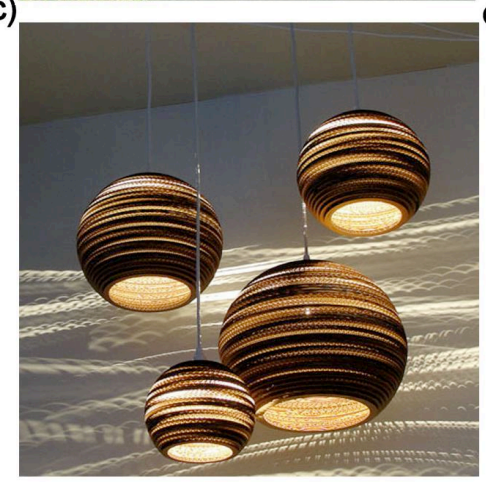

b)
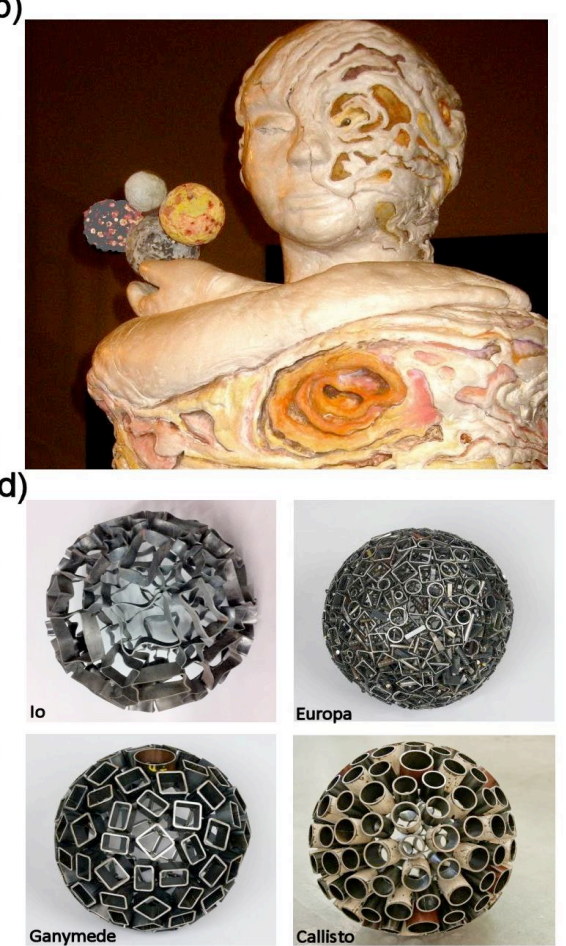

Figure 8. (a) Ganymede-Callisto Pod, toothpick sculpture by Franceska McCullough; (b) Jupiter, from The Planet Series, ceramic (2008), by Paula Rice; (c) Moons of Jupiter Scrap Lights, from repurposed cardboard by Graypants, Inc., Seattle (WA); (d) Moons of Jupiter (2007ongoing), steel, by Mark Castator, Boulder (CO)

\section{Final Thoughts}

Due to his artistic skills learned prior to the advent of the telescope, Galileo was able to offer realistic portrayals of the Moon's features that had been previously hidden to the un-aided eye. ${ }^{15}$ The discovery of Jupiter's moons presented a challenge to the cosmology of the day, but not to the descriptive aspects of those bodies. Their very existence was revolutionary, not the symbols (stars or later disks) he used to represent them. In the 400 years since the publication of Sidereus Nuncius, those

15 Samuel Y. Edgerton, 'The Function of Artistic Form in the Study of the Stars', in Stacy McCarroll (ed.), Celestial Images: Antiquarian Astronomical Charts and Maps from the Mendillo Collection. 
moons were given substance artistically in paintings and sculpture, and used as business logos and as names of commercial products. One can only imagine what Galileo's response would be today. I suspect he might be pleased as an artist, but upset about the lack of royalties from the commercial uses.

Acknowledgements. I am grateful for bringing to my attention various artistic uses of the Galilean moons by Inga Soderlund, Enrico Maria Corsini, Giles Davison, Keith Noll, Joei Wroten and Cesare Barbieri. Ms.Wroten's expert help with the manuscript and figure preparation is gratefully acknowledged. The INSAP-7 meeting organized by Nick Campion and colleagues offered inspiration and encouragement for this study. Sabbatical year support from Boston University and its Centre for Space Physics provided the time and resources that made this project possible. 